\title{
Determinants of sports, cycling, walking and overall leisure-time physical activity among postmenopausal women in Germany
}

\author{
Karen Steindorf ${ }^{1, *}$, Jenny Chang-Claude ${ }^{2}$, Dieter Flesch-Janys ${ }^{3}$ and Martina E Schmidt ${ }^{1}$ \\ 'Unit of Environmental Epidemiology, German Cancer Research Centre, Deutsches Krebsforschungszentrum, Im \\ Nevenheimer Feld 280, 69120 Heidelberg, Germany: ${ }^{2}$ Department of Cancer Epidemiology, German Cancer \\ Research Centre, Im Neuenheimer Feld 280, Heidelberg, Germany: ${ }^{3}$ Department of Medical Biometry and \\ Epidemiology, University Medical Center Hamburg-Eppendorf, Martinistrasse 52, Hamburg, Germany
}

Submitted 29 October 2009: Accepted 1 June 2010: First published online 13 July 2010

\begin{abstract}
Objective: Convincing evidence exists for a beneficial effect of physical activity (PA) on health and well-being for elderly women. Nevertheless, many women in Germany are insufficiently physically active. Activity promotion programmes should target women in particular need. Thus, we examined subject-related determinants of PA for postmenopausal women in Germany.

Design: Associations of sociodemographic, anthropometric, lifestyle and healthrelated factors with activity since the age of 50 years were assessed with multiple linear and logistic regression models, regarding overall leisure-time PA (LPA) in metabolic equivalent hours per week, engagement in sports (ever $v$. never), cycling (yes $v$. no) and walking ( $\geq 3.5 v .<3.5 \mathrm{~h} /$ week).

Setting: All controls from a population-based case-control study carried out in 2002-2005 in Germany (MARIE study).

Subjects: A total of 6569 postmenopausal women.

Results: We found significant associations of overall LPA, sports, cycling and walking in postmenopausal years with several characteristics, among others: obese $v$. normal BMI (adjusted OR: $\mathrm{OR}_{\text {sports }}=0 \cdot 73 ; \mathrm{OR}_{\text {cycling }}=0 \cdot 60 ; \mathrm{OR}_{\text {walking }}=0 \cdot 63$ ), tall $v$. short $\left(\mathrm{OR}_{\text {sports }}=1 \cdot 49\right)$, worker $v$. medium employee $\left(\mathrm{OR}_{\text {sports }}=0 \cdot 47\right)$, working full time $v$. unemployed $/$ retired $\left(\mathrm{OR}_{\text {sports }}=0 \cdot 80 ; \mathrm{OR}_{\text {walking }}=0 \cdot 56\right)$, current $v$. non-smoker $\left(\mathrm{OR}_{\text {sports }}=0.62 ; \quad \mathrm{OR}_{\text {cycling }}=0.62 ; \quad \mathrm{OR}_{\text {walking }}=0 \cdot 82\right), \quad$ non-German nationality $\left(\mathrm{OR}_{\text {sports }}=0 \cdot 58 ; \mathrm{OR}_{\text {cycling }}=0 \cdot 41\right)$. Parity seemed more relevant than marital status for sports or cycling activity. Further, CVD, hypertension and diabetes were significantly associated with lower activity $\left(\mathrm{OR}_{\text {sports }}=0.77,0.79\right.$ and $0.80 ; \mathrm{OR}_{\text {cycling }}=$ $0 \cdot 80,0 \cdot 75$ and $0 \cdot 85$, respectively).

Conclusions: Our results suggest potential target groups for promoting sports, cycling or walking activity. Postmenopausal women with chronic diseases may need to be stronger encouraged by their physicians to engage in adequate PA for the management of their diseases.
\end{abstract}

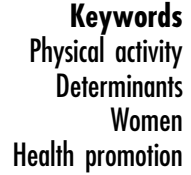

As in other developed countries, life expectancy continues to increase in Germany. The number of elderly Germans ( $\geq 65$ years) is expected to grow by about $50 \%$ between 2007 and 2030: from 16.5 million to about 24 million $^{(1)}$. Of these about $58 \%$ are women. Correspondingly, the prevalence of several diseases and disorders will increase substantially. Yet, regular physical activity (PA) may reduce the risks of many common health problems in the elderly such as CVD and type 2 diabetes $^{(2)}$, obesity, osteoporosis ${ }^{(3)}$, dementia $^{(4)}$, postmenopausal breast cancer and colon cancer $^{(5)}$, may maintain independent living ${ }^{(6)}$ and prevent falls $^{(7)}$. Despite this knowledge, a large proportion of elderly women appear to be still physically little active ${ }^{(8)}$. Activity campaigns or fitness courses apparently tend to reach the health-conscious women but not the subgroups in particular need ${ }^{(9)}$. Besides sports and exercise, day-to-day walking and cycling can contribute substantially to an active healthy lifestyle. Knowledge of the individual characteristics of women not engaging in those activities will be important to design effective specifically tailored interventions. Only a few studies have investigated activity determinants in the German population ${ }^{(8,10,11)}$, and none of those evaluated factors associated specifically with walking and cycling. Several studies have separately investigated demographic, lifestyle or psychosocial determinants of activity ${ }^{(8,10,12-16)}$, but few have comprehensively investigated a broad range of factors of interest simultaneously controlling for potentially confounding variables.

Thus, we examined the associations of a large number of sociodemographic, anthropometric, lifestyle and 
health-related individual characteristics with different PA types including sports, cycling and walking in a large, population-based sample of 6569 postmenopausal women in Germany, hereby taking potential confounding, interaction and collinearity into account.

\section{Methods}

\section{Study population}

These analyses comprised all postmenopausal female residents aged $50-75$ years from a population registries-based random sample who participated as controls in a large case-control study on breast cancer (MARIE study, Mammary carcinoma Risk factor Investigation). The study was carried out in 2002-2005 in two study regions in Germany: (i) the city and state of Hamburg in northern Germany (1.8 million inhabitants) and (ii) the Rhine-Neckar-Karlsruhe region in southern Germany ( $2 \cdot 8$ million inhabitants). Controls were randomly drawn from lists of residents provided by the population registries and frequency matched by birth year and study region to the breast cancer cases. Further details are published elsewhere ${ }^{(17)}$. The study was approved by the ethics committees of both the Universities of Heidelberg and Hamburg. All study participants gave written informed consent. A total of $7421(43 \cdot 4 \%)$ controls participated, of whom 6657 were postmenopausal and 6569 had data on PA since the age of 50 years.

\section{Data assessment}

Each participant was interviewed once face-to-face by trained interviewers using a standardised questionnaire to elicit comprehensive information on sociodemographic, lifestyle and health-related factors. Women were defined as postmenopausal if they reported a natural menopause 12 months before the reference date, a bilateral oophorectomy or cessation of menses due to radiation or chemotherapy. Those above 55 years of age whose menopausal status was unclear because of hysterectomy or hormone use were also considered as postmenopausal but assigned an unknown age at menopause. PA was assessed for the age periods of $30-49$ and $\geq 50$ years using an interviewadministered questionnaire ${ }^{(18)}$. Splitting a typical week into weekdays and weekends, each woman reported the number of hours per week separately for walking and cycling for this period. For exercise, participants were asked to list up to three sports performed during this age period, including type, duration, frequency and number of years. A metabolic equivalent (MET) value was assigned to each reported activity ${ }^{(19,20)}$. Leisure-time PA (LPA) in MET hours per week $($ MET $\times$ h/week) was calculated by summing the average hours per week spent walking, cycling and engaged in sports, weighted by the appropriate MET values $^{(21)}$. Since the various components of LPA, i.e. walking, cycling and sports, may be associated with different subject characteristics, we also investigated those activity types separately. As one-third of the study population never engaged in sports since the age of 50 years, and the other participants were mainly little or moderately active, we were interested in examining the determinants of women who engaged in sports compared with those who never engaged in sports. Hence, we created a binary sports variable (active/inactive). The same was applied for cycling (active/inactive). For walking also, we created a binary variable. As nearly all women spent some time in walking during the week, we chose a meaningful cut-off point of $3.5 \mathrm{~h} /$ week. This corresponds to half an hour of walking per day. Although time spent with occupational and household PA had also been assessed, investigating determinants for these PA variables would not yield reasonable results for public health recommendations, as there was no sufficient differentiation between tasks with different intensities.

\section{Statistical methods}

Associations between the continuous LPA variable and independent variables were assessed with multiple regression models. The dependent variable was log-transformed due to skewness. Collinearity diagnostics indicated no important violations among the variables in our model (all variance inflation factors were $<10$ ). For the binary PA variables multiple logistic regression analyses were used. All independent variables were entered into the logistic regression model simultaneously; thus the effect of a predictor was calculated after statistically controlling for all the others. All $P$ values were two-sided. Analyses were conducted using the SAS statistical software package version 9·1 (SAS Institute Inc., Cary, NC, USA).

As independent variables we investigated the following factors: year of birth (categorised), BMI at the age of 25-50 years based on self-reported weight and height $(<22.5$, $\left.22 \cdot 5 \leq 25 \cdot 0,25 \cdot 0 \leq 30 \cdot 0, \geq 30 \cdot 0 \mathrm{~kg} / \mathrm{m}^{2}\right)$, height $(\leq 155,>155-$ $165,>165-175,>175 \mathrm{~cm}$ ), education (basic, advanced, high), current or last occupation (never employed, worker, simple, medium, high employed, self-employed, civil servants), current working hours if ever employed, housework (h/week), marital status (married, widowed, divorced, separated, single), number of live-born children, age at first pregnancy, ever breast-fed, nationality, smoking (never, ex-smoker, current), alcohol (no, 1-18 g/d, $\geq 19 \mathrm{~g} / \mathrm{d}$ ) and coexisting diseases. Regarding occupational status, employees, who comprised the majority of women, were further separated into simple employees, who executed tasks upon instruction (such as clerks, typists), medium employees performing more self-dependent tasks (e.g. bookkeeper, technician) and high employees with advanced responsibilities or executive functions (e.g. supervisor, research assistant).

\section{Results}

The analyses included 6569 postmenopausal women with a median age of 63 (interquartile range: 59-67) years. The majority were married $(65 \cdot 5 \%)$, parous $(84 \cdot 2 \%)$, had 
Table 1 Distribution of different activities

\begin{tabular}{lrrrll}
\hline & Median & \multicolumn{1}{c}{ Q1 } & Q3 & Definition of being active & Classified as active (\%) \\
\hline Leisure-time PA (MET $\times$ h/week) & 43.59 & 26.00 & 69.58 & Continuous & - \\
Sports (h/week) & 0.57 & 0.00 & 1.96 & Any sports since the age of 50 years & $64 \cdot 85$ \\
Cycling (h/week) & $1 \cdot 17$ & 0.00 & 4.00 & Any cycling in a typical week & $58 \cdot 11$ \\
Walking (h/week) & 6.50 & 3.00 & 9.33 & Walking $\geq 3.5 \mathrm{~h} /$ week & $74 \cdot 17$ \\
Household tasks (h/week) & 24.00 & 16.00 & 35.00 & Continuous & - \\
\hline
\end{tabular}

Q1, first quartile; Q3, third quartile; PA, physical activity; MET, metabolic equivalent.

basic education (58.5\%) and were already retired (71.8\%) (Table 2). Their median leisure-time activity since the age of 50 years was $43.6 \mathrm{MET} \times \mathrm{h} /$ week (Table 1 ), hereby most time was spent walking (median $6.5 \mathrm{~h} /$ week), followed by cycling $(1 \cdot 2 \mathrm{~h} /$ week $)$ and rarely spent in sports $(0.6 \mathrm{~h} /$ week $)$. About one-third of the women were not engaged in any sports since the age of 50 years. There were no strong correlations between the variables sports and cycling $(r=0 \cdot 16)$ or walking $(r=0 \cdot 04)$, nor between cycling and walking $(r=0 \cdot 03)$.

In multiple regression models (Table 2), increased BMI was significantly associated with decreased LPA, sports and walking activity. With regard to cycling, only obesity $\left(\mathrm{BMI} \geq 30 \cdot 0 \mathrm{~kg} / \mathrm{m}^{2}\right)$ was associated with decreased activity. Interestingly, independent of BMI, body height was also associated with activity: taller women were more likely to engage in sports than shorter women. Short women also appeared to cycle less but walk more than medium-sized women; yet, there was no association with overall LPA.

Basic educational status was associated with low sports and high cycling activity only when occupational status and working hours were not considered (data not shown). Considering all three variables simultaneously, these associations of education with sports and cycling were less strong and non-significant. Sports was significantly associated with occupation, with civil servants being most likely engaged in any sports, followed by high employees, while simple employees, workers and never employed women were most likely to do no sports. Yet, civil servants and self-employed women were less likely to cycle, especially compared to working women. Selfemployment was also strongly associated with low walking activity, while high employees were most likely to walk $\geq 3.5 \mathrm{~h} /$ week. In addition, working time was associated with activity, showing less engagement in sports and walking for women working full time. In contrast, increased time spent on housework was associated with higher LPA, dominated by cycling.

For marital status and parity, no associations with overall LPA were found, but parity was positively associated with being engaged in sports and cycling with no difference by the number of live births. For marital status, no association with sports was found, but being widowed, compared to being married, decreased cycling activity. Interestingly, considering marital status without adjustment for parity showed significantly lower likelihoods for activity in singles compared to married women regarding sports $(\mathrm{OR}=0 \cdot 66)$ and cycling $(\mathrm{OR}=0 \cdot 58)$. However, including a combined variable into the model showed significantly higher likelihood for activity for parous ever married women compared to nulliparous singles (sports: OR $=1 \cdot 49$, 95\% CI $1 \cdot 15,1 \cdot 91$; cycling: $\mathrm{OR}=1 \cdot 82,95 \% \mathrm{CI} 1 \cdot 43,2 \cdot 32$ ), but there was no difference between singles and ever married nulliparous women (data not shown). Overall, parity seemed to be more relevant than marital status for sports or cycling activity. In contrast, walking activity was not associated with parity but with marital status. Widowed or divorced women were more likely to walk than married women. Among the parous women, however, those with early maternity were more likely to do no sports even at postmenopausal years compared to those women who had children at a later age. Further, parous women who had never breast-fed were significantly less likely to be active in sports or cycling, even adjusted for all other sociodemographic factors.

Non-German nationality was strongly associated with low levels of LPA $(P=0 \cdot 01)$, cycling $(\mathrm{OR}=0 \cdot 41)$ and sports $(\mathrm{OR}=0 \cdot 58)$ even after adjustment for other sociodemographic factors, but there was no association with walking.

Current smoking was a strongly significant determinant of low levels of activity with respect to all types of PA. Women who reported drinking no alcohol were less active in LPA and sports than women in the highest category.

Results for coexisting diseases, adjusted for all other factors, are shown in Table 3. Diabetes was associated with less activity for all activity types. Women diagnosed with CVD or hypertension engaged less in LPA, sports and cycling. More sports activity was reported by women with benign breast diseases, myoma and arthritis or rheumatism, less sports by women having any lung disease.

\section{Discussion}

Our study of 6569 postmenopausal women in Germany suggests potential target groups that may need particular consideration to promote sports as well as walking and cycling, such as overweight, obese or small women, full-time employees, workers, simple employees, selfemployed or housewives, current smokers, immigrants, 


\begin{tabular}{|c|c|c|c|c|c|c|c|c|c|}
\hline & \multirow[b]{3}{*}{$\% \ddagger$} & & & \multicolumn{6}{|c|}{ Odds ratio for being active } \\
\hline & & \multicolumn{2}{|c|}{ Leisure-time PA $\S(\mathrm{MET} \times \mathrm{h} /$ week) } & \multicolumn{2}{|c|}{ Sports (ever $v$. never) } & \multicolumn{2}{|c|}{ Cycling (yes v. no) } & \multicolumn{2}{|c|}{ Walking ( $\geq 3.5 \mathrm{v} .>3.5 \mathrm{~h} /$ week) } \\
\hline & & $\beta \|$ & $P$ & OR & $95 \% \mathrm{Cl}$ & OR & $95 \% \mathrm{Cl}$ & OR & $95 \% \mathrm{Cl}$ \\
\hline \multicolumn{10}{|l|}{ Year of birth } \\
\hline $1926-1934$ & $17 \cdot 26$ & 0.000 & - & 1.00 & - & 1.00 & - & 1.00 & - \\
\hline 1935-1939 & $30 \cdot 52$ & -0.004 & 0.9184 & $1 \cdot 13$ & $0.96,1 \cdot 33$ & 1.07 & $0.92,1.25$ & 0.91 & $0 \cdot 76,1 \cdot 10$ \\
\hline 1940-1944 & $29 \cdot 96$ & 0.062 & $0 \cdot 1473$ & 1.06 & $0 \cdot 89,1 \cdot 26$ & $1 \cdot 19$ & $1 \cdot 01,1 \cdot 40^{\star}$ & 0.96 & $0 \cdot 78,1 \cdot 17$ \\
\hline 1945-1949 & $17 \cdot 57$ & 0.064 & 0.2293 & 1.09 & $0 \cdot 87,1 \cdot 35$ & $1 \cdot 11$ & $0 \cdot 90,1 \cdot 36$ & $0 \cdot 86$ & $0 \cdot 68,1 \cdot 10$ \\
\hline 1950-1955 & $4 \cdot 69$ & 0.035 & 0.6477 & 0.92 & $0.67,1.25$ & $1 \cdot 13$ & $0 \cdot 84,1 \cdot 52$ & 0.95 & $0.68,1 \cdot 33$ \\
\hline \multicolumn{10}{|l|}{ BMl at the age of $25-50$ years } \\
\hline Low & $44 \cdot 22$ & 0.074 & 0.0171 & $1 \cdot 14$ & $1 \cdot 01,1 \cdot 30^{\star}$ & 0.95 & $0.84,1 \cdot 07$ & $1 \cdot 10$ & $0.96,1 \cdot 27$ \\
\hline Normal & $31 \cdot 22$ & 0.000 & - & $1 \cdot 00$ & - & $1 \cdot 00$ & - & $1 \cdot 00$ & - \\
\hline Overweight & $20 \cdot 44$ & -0.076 & 0.0445 & $0 \cdot 86$ & $0 \cdot 74,1 \cdot 00$ & 0.96 & $0 \cdot 83,1 \cdot 11$ & 0.84 & $0.71,0.99^{\star}$ \\
\hline Obese & 4.05 & -0.443 & $<0.0001$ & 0.73 & $0.55,0.96^{\star}$ & 0.60 & $0.46,0.79^{\star \star}$ & 0.63 & $0.47,0.85^{\star \star}$ \\
\hline \multicolumn{10}{|l|}{ Height (cm) } \\
\hline$\leq 155$ & $6 \cdot 52$ & 0.000 & - & $1 \cdot 00$ & - & $1 \cdot 00$ & - & $1 \cdot 00$ & - \\
\hline$>155-165$ & $52 \cdot 31$ & 0.063 & 0.2519 & $1 \cdot 22$ & $0.99,1.52$ & $1 \cdot 31$ & $1 \cdot 06,1 \cdot 62^{\star}$ & $0 \cdot 86$ & $0 \cdot 67,1.09$ \\
\hline$>165-175$ & 38.03 & 0.063 & 0.2659 & $1 \cdot 26$ & $1 \cdot 01,1 \cdot 58^{\star}$ & $1 \cdot 31$ & $1 \cdot 05,1 \cdot 62^{\star}$ & 0.73 & $0.57,0.94^{\star}$ \\
\hline$>175$ & $3 \cdot 15$ & 0.020 & 0.8250 & 1.49 & $1 \cdot 02,2 \cdot 19^{\star}$ & $1 \cdot 12$ & $0 \cdot 79,1 \cdot 58$ & 0.91 & $0 \cdot 59,1 \cdot 40$ \\
\hline \multicolumn{10}{|l|}{ Education } \\
\hline Basic & $58 \cdot 50$ & -0.098 & 0.0500 & $0 \cdot 86$ & $0 \cdot 70,1 \cdot 06$ & 1.05 & $0 \cdot 87,1 \cdot 27$ & $0 \cdot 82$ & $0.65,1.03$ \\
\hline Advanced & 28.01 & 0.028 & 0.5631 & $1 \cdot 10$ & $0 \cdot 89,1 \cdot 35$ & $1 \cdot 07$ & $0 \cdot 88,1 \cdot 29$ & $1 \cdot 00$ & $0 \cdot 79,1 \cdot 26$ \\
\hline High & $13 \cdot 49$ & 0.000 & - & $1 \cdot 00$ & - & $1 \cdot 00$ & - & $1 \cdot 00$ & - \\
\hline \multicolumn{10}{|l|}{ Current or last occupation } \\
\hline Never employed & $1 \cdot 49$ & -0.357 & 0.0013 & 0.59 & $0.38,0.91^{*}$ & 0.94 & $0.61,1 \cdot 45$ & 0.61 & $0.39,0.97^{\star}$ \\
\hline Worker & $13 \cdot 67$ & 0.041 & 0.3707 & 0.47 & $0.40,0.57^{\star *}$ & $1 \cdot 13$ & $0.94,1.35$ & $1 \cdot 03$ & $0 \cdot 84,1 \cdot 25$ \\
\hline Simple employed & $22 \cdot 32$ & 0.023 & 0.5371 & 0.78 & $0.67,0.91^{\star \star}$ & 1.04 & $0.90,1 \cdot 21$ & 0.92 & $0.77,1.09$ \\
\hline Medium employed & $33 \cdot 35$ & 0.000 & - & $1 \cdot 00$ & - & $1 \cdot 00$ & - & $1 \cdot 00$ & - \\
\hline High employed & $15 \cdot 88$ & 0.066 & $0 \cdot 1058$ & $1 \cdot 12$ & $0.94,1.33$ & 0.95 & $0 \cdot 81,1 \cdot 11$ & $1 \cdot 26$ & $1 \cdot 03,1 \cdot 54^{\star}$ \\
\hline Self-employed & $7 \cdot 87$ & $-0 \cdot 300$ & $<0.0001$ & 0.90 & $0 \cdot 72,1 \cdot 12$ & 0.74 & $0.60,0.91^{\star \star}$ & 0.48 & $0 \cdot 39,0 \cdot 60^{\star \star}$ \\
\hline Civil servant & $5 \cdot 22$ & $-0 \cdot 136$ & 0.0523 & $1 \cdot 34$ & $0 \cdot 98,1 \cdot 84$ & 0.82 & $0 \cdot 63,1 \cdot 07$ & $0 \cdot 82$ & $0.59,1.13$ \\
\hline \multicolumn{10}{|c|}{ Current working hours (if ever employed) } \\
\hline $0 \mathrm{~h} /$ week; retired & $71 \cdot 81$ & 0.000 & - & $1 \cdot 00$ & - & $1 \cdot 00$ & - & $1 \cdot 00$ & - \\
\hline 1-14 h/week & $5 \cdot 05$ & 0.093 & $0 \cdot 1270$ & 1.45 & $1 \cdot 11,1 \cdot 90^{\star \star}$ & $1 \cdot 20$ & $0.94,1.53$ & 0.90 & $0 \cdot 68,1 \cdot 18$ \\
\hline 15-34 h/week & $9 \cdot 91$ & -0.073 & $0 \cdot 1373$ & $1 \cdot 11$ & $0 \cdot 89,1 \cdot 37$ & $1 \cdot 12$ & $0.92,1.37$ & 0.83 & $0 \cdot 67,1 \cdot 04$ \\
\hline$\geq 35 \mathrm{~h} /$ week & $11 \cdot 74$ & -0.286 & $<0.0001$ & 0.80 & $0.65,0.98^{\star}$ & $1 \cdot 01$ & $0 \cdot 83,1 \cdot 22$ & 0.56 & $0.45,0.69^{\star \star}$ \\
\hline \multicolumn{10}{|l|}{ Housework (h/week) } \\
\hline Continuous, per $10 \mathrm{~h} /$ week & & 0.021 & 0.0263 & 0.97 & $0.94,1 \cdot 01$ & 1.09 & $1 \cdot 05,1 \cdot 13^{\star \star}$ & 0.99 & $0.95,1.03$ \\
\hline \multicolumn{10}{|l|}{ Marital status } \\
\hline Married & $65 \cdot 47$ & 0.000 & - & 1.00 & - & $1 \cdot 00$ & - & 1.00 & - \\
\hline Widowed & $16 \cdot 32$ & 0.024 & 0.5249 & 0.95 & $0 \cdot 82,1 \cdot 11$ & 0.80 & $0.69,0.92^{\star \star}$ & $1 \cdot 25$ & $1 \cdot 06,1 \cdot 48^{\star \star}$ \\
\hline Divorced & $11 \cdot 43$ & 0.026 & 0.5568 & $0 \cdot 89$ & $0 \cdot 75,1 \cdot 07$ & 0.98 & $0 \cdot 83,1 \cdot 16$ & $1 \cdot 32$ & $1 \cdot 07,1 \cdot 63^{\star \star}$ \\
\hline Separated & $1 \cdot 28$ & $0 \cdot 149$ & $0 \cdot 2011$ & 0.85 & $0.53,1.36$ & 0.94 & $0 \cdot 60,1 \cdot 48$ & $1 \cdot 62$ & $0.89,2.93$ \\
\hline Single & $5 \cdot 48$ & -0.020 & 0.7578 & $0 \cdot 83$ & $0.64,1.08$ & $0 \cdot 85$ & $0 \cdot 66,1 \cdot 10$ & $1 \cdot 10$ & $0 \cdot 81,1 \cdot 51$ \\
\hline \multicolumn{10}{|l|}{ Number of live-born children } \\
\hline 0 & $15 \cdot 82$ & 0.000 & - & $1 \cdot 00$ & - & $1 \cdot 00$ & - & $1 \cdot 00$ & - \\
\hline 1 & $25 \cdot 21$ & 0.079 & $0 \cdot 1721$ & 1.53 & $1 \cdot 20,1 \cdot 94^{\star \star}$ & $1 \cdot 28$ & $1 \cdot 02,1 \cdot 59^{\star}$ & 1.01 & $0 \cdot 77,1 \cdot 32$ \\
\hline 2 & $38 \cdot 39$ & 0.080 & $0 \cdot 1795$ & 1.89 & $1 \cdot 47,2 \cdot 43^{\star \star}$ & 1.45 & $1 \cdot 15,1 \cdot 82^{\star \star}$ & $0 \cdot 84$ & $0 \cdot 64,1 \cdot 11$ \\
\hline$\geq 3$ & $20 \cdot 58$ & -0.030 & 0.6382 & 1.65 & $1 \cdot 25,2 \cdot 16^{\star \star}$ & $1 \cdot 38$ & $1 \cdot 07,1 \cdot 77^{\star}$ & 0.74 & $0.55,1.00^{\star}$ \\
\hline
\end{tabular}


Table 2 Continued

\begin{tabular}{|c|c|c|c|c|c|c|c|c|c|}
\hline & \multirow[b]{3}{*}{$\% \ddagger$} & & & \multicolumn{6}{|c|}{ Odds ratio for being active } \\
\hline & & \multicolumn{2}{|c|}{ Leisure-time PA§ (MET $\times \mathrm{h} /$ week $)$} & \multicolumn{2}{|c|}{ Sports (ever v. never) } & \multicolumn{2}{|c|}{ Cycling (yes v. no) } & \multicolumn{2}{|c|}{ Walking ( $\geq 3.5 \mathrm{v} .>3.5 \mathrm{~h} /$ week) } \\
\hline & & $\beta \|$ & $P$ & OR & $95 \% \mathrm{Cl}$ & OR & $95 \% \mathrm{Cl}$ & OR & $95 \% \mathrm{Cl}$ \\
\hline \multicolumn{10}{|c|}{ If parous: age at first birth (years) } \\
\hline $14-19$ & $9 \cdot 68$ & -0.028 & 0.6499 & 0.66 & $0.51,0.86^{\star \star}$ & $1 \cdot 04$ & $0 \cdot 82,1 \cdot 33$ & 0.98 & $0 \cdot 75,1 \cdot 29$ \\
\hline $20-24$ & $37 \cdot 45$ & 0.011 & 0.8202 & 0.74 & $0.60,0.91^{\star \star}$ & 1.03 & $0 \cdot 86,1 \cdot 24$ & $1 \cdot 12$ & $0.90,1.39$ \\
\hline $25-29$ & $25 \cdot 67$ & 0.017 & $0 \cdot 7172$ & 0.87 & $0 \cdot 71,1 \cdot 07$ & $1 \cdot 11$ & $0.92,1.33$ & $1 \cdot 25$ & $1 \cdot 00,1 \cdot 55^{\star}$ \\
\hline$\geq 30$ & $11 \cdot 39$ & 0.000 & - & $1 \cdot 00$ & - & $1 \cdot 00$ & - & $1 \cdot 00$ & - \\
\hline \multicolumn{10}{|c|}{ If parous: breast-fed } \\
\hline Ever & $66 \cdot 60$ & 0.000 & - & 1.00 & - & 1.00 & - & 1.00 & - \\
\hline Never & $17 \cdot 58$ & -0.029 & 0.4131 & 0.75 & $0 \cdot 65,0.87^{\star \star}$ & 0.83 & $0.72,0.95^{\star *}$ & $0 \cdot 90$ & $0 \cdot 77,1 \cdot 06$ \\
\hline \multicolumn{10}{|c|}{ German nationality } \\
\hline Yes & $98 \cdot 20$ & 0.000 & - & 1.00 & - & 1.00 & - & $1 \cdot 00$ & - \\
\hline No & $1 \cdot 80$ & -0.325 & 0.0012 & 0.58 & $0.39,0.87^{\star \star}$ & 0.41 & $0 \cdot 28,0 \cdot 61^{\star *}$ & 0.98 & $0 \cdot 64,1 \cdot 49$ \\
\hline \multicolumn{10}{|l|}{ Smoking } \\
\hline Never & $52 \cdot 79$ & 0.000 & - & 1.00 & - & 1.00 & - & $1 \cdot 00$ & - \\
\hline Ex & $28 \cdot 74$ & 0.017 & 0.5942 & $1 \cdot 20$ & $1 \cdot 05,1 \cdot 37^{\star \star}$ & 0.97 & $0.86,1 \cdot 09$ & $0 \cdot 96$ & $0 \cdot 84,1 \cdot 11$ \\
\hline Current & $18 \cdot 42$ & $-0 \cdot 217$ & $<0.0001$ & 0.62 & $0.53,0.72^{\star \star}$ & 0.62 & $0.54,0.71^{\star *}$ & 0.82 & $0.69,0.97^{*}$ \\
\hline \multicolumn{10}{|c|}{ Alcohol $(g / d)$} \\
\hline No & $14 \cdot 31$ & $-0 \cdot 140$ & 0.0070 & 0.60 & $0.48,0.73^{\star \star}$ & 0.96 & $0 \cdot 79,1 \cdot 18$ & $0 \cdot 88$ & $0 \cdot 70,1 \cdot 10$ \\
\hline $1-18$ & $72 \cdot 81$ & -0.003 & 0.9348 & 0.96 & $0 \cdot 81,1 \cdot 14$ & $1 \cdot 15$ & $0.99,1.35$ & 1.03 & $0 \cdot 86,1 \cdot 24$ \\
\hline$\geq 19$ & $12 \cdot 74$ & 0.000 & - & 1.00 & - & 1.00 & - & $1 \cdot 00$ & - \\
\hline
\end{tabular}

PA, physical activity; MET, metabolic equivalent.

${ }^{*} 0.01 \leq P<0.05,{ }^{* *} P<0.01$.

+Models include all variables presented in Tables 2 and 3 simultaneously.

¥Percentages based on the whole cohort ( $n$ 6569).

\&Leisure-time PA is calculated as the sum of MET*h/week spent in sports, cycling and walking.

IILinear regression; $R^{2}=0 \cdot 10$. 
Table 3 Results of multiple regression modelst on determinants for different PA types: coexisting diseases

\begin{tabular}{|c|c|c|c|c|c|c|c|c|c|}
\hline \multirow[b]{3}{*}{ Yes $v$. no } & \multirow{3}{*}{$\begin{array}{l}\text { Women } \\
\text { with } \\
\text { disease } \\
(\%) \ddagger\end{array}$} & \multirow{2}{*}{\multicolumn{2}{|c|}{ Leisure-time $\mathrm{PA} \S(\mathrm{MET} \times \mathrm{h} /$ week $)$}} & \multicolumn{6}{|c|}{ Odds ratio for being active } \\
\hline & & & & \multicolumn{2}{|c|}{ Sports (ever $v$. never) } & \multicolumn{2}{|c|}{ Biking (yes v. no) } & \multicolumn{2}{|c|}{ Walking ( $\geq 3.5 \mathrm{v} .>3.5 \mathrm{~h} /$ week $)$} \\
\hline & & $\beta \S$ & $P$ & OR & $95 \% \mathrm{Cl}$ & OR & $95 \% \mathrm{Cl}$ & OR & $95 \% \mathrm{Cl}$ \\
\hline Hypertension & $41 \cdot 32$ & -0.060 & 0.0315 & 0.79 & $0 \cdot 70,0 \cdot 88^{\star \star}$ & 0.85 & $0 \cdot 76,0.95^{\star \star}$ & $1 \cdot 01$ & $0 \cdot 89,1 \cdot 14$ \\
\hline Diabetes & $7 \cdot 37$ & $-0 \cdot 118$ & 0.0237 & 0.80 & $0.65,0.98^{\star}$ & 0.75 & $0.62,0.92^{\star \star}$ & 0.75 & $0.60,0.93$ \\
\hline CVD & $14 \cdot 54$ & $-0 \cdot 110$ & 0.0041 & 0.77 & $0.66,0.90^{\star *}$ & 0.80 & $0.69,0.93^{\star *}$ & 1.04 & $0.88,1 \cdot 24$ \\
\hline Deep vein thrombosis & $7 \cdot 26$ & -0.085 & 0.0945 & 0.90 & $0 \cdot 73,1 \cdot 10$ & 0.88 & $0 \cdot 72,1 \cdot 07$ & $1 \cdot 01$ & $0 \cdot 81,1 \cdot 28$ \\
\hline Chronic lung diseases & $10 \cdot 43$ & -0.042 & 0.3333 & 0.84 & $0.71,1.00^{\star}$ & 0.99 & $0 \cdot 84,1 \cdot 17$ & 0.91 & $0 \cdot 75,1 \cdot 10$ \\
\hline Chronic liver diseases & $4 \cdot 13$ & 0.055 & 0.4068 & 1.01 & $0 \cdot 77,1 \cdot 32$ & 0.90 & $0 \cdot 69,1 \cdot 16$ & $1 \cdot 15$ & $0.85,1.56$ \\
\hline $\begin{array}{l}\text { Chronic bowel, bladder or kidney } \\
\text { diseases }\end{array}$ & $11 \cdot 57$ & -0.051 & $0 \cdot 2167$ & $1 \cdot 07$ & $0 \cdot 90,1 \cdot 26$ & 0.91 & $0 \cdot 78,1 \cdot 07$ & 0.90 & $0.75,1.09$ \\
\hline Thyroid disease & $30 \cdot 10$ & 0.026 & $0 \cdot 3698$ & 1.04 & $0 \cdot 92,1 \cdot 16$ & 1.02 & $0.91,1 \cdot 14$ & $1 \cdot 11$ & $0 \cdot 98,1 \cdot 27$ \\
\hline Migraine & $23 \cdot 05$ & -0.037 & $0 \cdot 2414$ & 0.94 & $0 \cdot 83,1 \cdot 07$ & 0.90 & $0 \cdot 80,1 \cdot 01$ & $1 \cdot 06$ & $0 \cdot 92,1 \cdot 22$ \\
\hline Ovarian cysts & $15 \cdot 06$ & 0.073 & 0.0485 & $1 \cdot 02$ & $0 \cdot 88,1 \cdot 19$ & $1 \cdot 08$ & $0.94,1.25$ & $1 \cdot 14$ & $0.96,1.36$ \\
\hline Myoma & $34 \cdot 16$ & 0.019 & 0.4835 & $1 \cdot 12$ & $1 \cdot 00,1 \cdot 26$ & 0.97 & $0.87,1.08$ & 0.96 & $0.85,1.09$ \\
\hline Family history of breast cancer & $11 \cdot 68$ & 0.006 & $0 \cdot 8852$ & $1 \cdot 18$ & $0.99,1.39$ & $1 \cdot 12$ & $0.95,1 \cdot 31$ & 0.92 & $0 \cdot 77,1 \cdot 10$ \\
\hline Benign breast disease & $33 \cdot 89$ & 0.0004 & 0.9876 & $1 \cdot 14$ & $1 \cdot 01,1 \cdot 28^{\star}$ & 1.03 & $0.92,1 \cdot 15$ & 0.94 & $0.83,1.07$ \\
\hline Arthritis, rheumatism & $52 \cdot 42$ & -0.013 & $0 \cdot 6187$ & $1 \cdot 12$ & $1 \cdot 00,1 \cdot 25^{\star}$ & $1 \cdot 01$ & $0.91,1 \cdot 12$ & 0.98 & $0 \cdot 87,1 \cdot 11$ \\
\hline Osteoporosis & $11 \cdot 78$ & -0.029 & 0.4878 & $0 \cdot 89$ & $0 \cdot 76,1 \cdot 05$ & 0.88 & $0 \cdot 75,1 \cdot 04$ & 0.90 & $0 \cdot 75,1 \cdot 08$ \\
\hline
\end{tabular}

PA, physical activity; MET, metabolic equivalent.

${ }^{*} 0.01 \leq P<0.05,{ }^{* *} P<0.01$.

tModels include all variables presented in Tables 2 and 3 simultaneously.

$\ddagger$ Percentages based on the whole cohort ( $n$ 6569).

$\S$ Leisure-time PA is calculated as the sum of MET*h/week spent in sports, cycling and walking.

IILinear regression; $R^{2}=0 \cdot 10$. 
nulliparous women or women with early maternity. Furthermore, women with diabetes, CVD or hypertension were less likely to be physically active, although those women could strongly benefit from PA for the management of their diseases.

\section{Strength and limitations}

One strength of our study is the comprehensive assessment of anthropometric, sociodemographic, lifestyle and health-related factors specifically tailored for women. Thus, we assessed the so far rarely investigated determinants that might be important for activity behaviour, specifically in women, such as parity, employment pattern or housework. In contrast to most previous studies, we examined determinants of activity simultaneously, taking potential confounding, interaction and collinearity into account. Furthermore, LPA was assessed in detail, considering different components of activity such as sports, cycling and walking, which provides more differentiated results than most previous studies. Besides sports and exercise, day-to-day walking and cycling can contribute substantially to an active healthy lifestyle. Although overall LPA might be most relevant for health, the results differentiated by type of activity may be of greater value for public health, because they indicate specific domains with need for action. While in some target groups LPA could be (further) enhanced by sports promotion programmes, in other subgroups promoting everyday cycling or walking may be needed. Finally, the behavioural pattern cannot easily be transferred from one country to another. Our study adds to the scarce knowledge on activity determinants in Germany, e.g. providing insight into the activity behaviour related to different occupational patterns, activity behaviours of immigrants and of women with chronic diseases. In particular, this is the first study that evaluated factors associated specifically with walking and cycling in Germany, which are common types of activity, especially in this country.

A limitation of our study is the reliance on self-reported recall of $\mathrm{PA}$ and the limited evaluation of psychometric properties of the questionnaire. The validity was evaluated by comparison with a distinct questionnaire structured by the intensity of PA. Since the questionnaire aimed to assess PA of the distant past, a validation against activity diaries, accelerometers or doubly labelled water technique was not feasible. We cannot fully exclude the possibility that there might be over- or under-reporting of activities among some population subgroups. However, while the total amount of time spent in LPA might be imprecisely assessed, women likely recalled well whether or not they were engaged in sports or cycling. Hence the binary variables for sports and cycling may be more precise. Further, the considered age period of $\geq 50$ years covered for some women a wide lifetime period, potentially introducing poor recollection and over- or under-reporting of the true activity levels. These measurement errors might have distorted the true effects towards the null. In addition, the study population may not be fully representative of the German population, since the study participants were recruited as controls in a matched case-control study on breast cancer. In addition, response rates $(43.4 \%)$ were low, potentially introducing selection bias. However, it is not an aim of the study to provide prevalence of activity, but rather to investigate factors associated with the given PA pattern. It is unlikely that the observed associations have been substantially affected by a potential selection of women. Nevertheless, our proportion of women who did not engage in any sports $(35 \%)$ is similar to results from a population-based health survey in Germany (about 37\%) ${ }^{(8)}$ and another health survey in Baden-Wuerttemberg, Germany (about $37 \%)^{(10)}$. Thus, the observed skewness in the LPA variable in our study is in accordance with other German data and is explained partly by a high proportion of women inactive with respect to cycling and sports.

\section{Associations between physical activity and different characteristics}

Consistent with results from other studies ${ }^{(10,12,13,15)}$, we found an inverse association between BMI and all activity variables, except cycling in which only obese women $\left(\mathrm{BMI} \geq 30 \cdot 0 \mathrm{~kg} / \mathrm{m}^{2}\right)$ were significantly less engaged. In contrast to previous studies, we were able to consider the typical BMI in premenopausal years, i.e. BMI before the age period for which PA was investigated. A high BMI could be a consequence of a low level of PA. However, obesity in premenopausal years remained a significant determinant for lower LPA in postmenopausal years even after adjustment for LPA in premenopausal years. Hence, being obese may hinder women from being physically active, independent of their activity behaviour in younger years. Some participants reported being ashamed about their appearance, or were afraid of being unable to keep up with others in a sports group as reasons for no engagement in sports. This is in line with a recent study that found that overweight perception may be a barrier to PA participation ${ }^{(22)}$. As cycling appeared to be as common in overweight women as in normal weight women, campaigns aiming at increasing cycling activity might reach overweight women who do not like to engage in other sports.

Interestingly, body height was an independent determinant for sports activity, with and without (data not shown) adjustment for BMI. Women at $\leq 155 \mathrm{~cm}$ of height had $49 \%$ higher odds of being inactive than women taller than $175 \mathrm{~cm}$. The majority $(77 \%)$ of inactive short women were already not engaged in sports in younger years. Possibly, short women had less feelings of success in sports during adolescence and therefore were less motivated to engage in sports later on. We are not aware of any other study that has investigated body height as a possible determinant of activity behaviour. Taking those results together, one could speculate whether courses 
explicitly offered for 'beginners', who so far felt not sportive enough to go to a regular sports group, could motivate these women to overcome their inhibitions. As in other studies ${ }^{(8,10,13,14,23)}$ current smoking was associated with a higher likelihood of having a low level of activity, regarding sports, walking and cycling, as well as overall LPA. Smoking might in part reflect lower health consciousness. Ex-smokers showed similar activity levels to non-smokers for LPA, walking and cycling, but they were more likely to engage in sports than never or current smokers in our study. The effect of smoking was independent of the effect of potentially smoking-related chronic diseases. Nevertheless, it is possible that smoking itself may hinder women from sports, cycling or walking through negative short-term health effects, such as bronchospasm. Physicians may need to intensify promotion of PA in smokers, e.g. by placing them into a walking group.

PA has been related with social status in some, but not all studies $^{(8,16,24)}$. In our study, lower educational status was related with low sport activity. However, after full adjustment, occupational status appeared to be more relevant than educational status. As the German school system for women in the birth cohort that was considered comprised 8- or 9-year elementary school, education does not strongly reflect socio-economic status in these postmenopausal women. Simple employees, workers, never employed women (covering primarily housewives) and women working full time were most likely to do no sports. In contrast, civil servants (primarily teachers) and self-employed women were less likely to cycle compared to the other occupational groups, especially the working women. More domain- and typespecific studies might help to tailor activity-promoting initiatives to specific working situations.

Non-Germans were more likely to be at a low level of PA compared to women with German nationality. It is noteworthy that only 118 women (2\%) reported non-German nationality in our study population, probably due to the restriction conferred by the personal interview in German language. There are likely more immigrants, especially from Eastern Europe, who now have German nationality. Nevertheless, we could identify some differences between the nationalities. Among women from the USA, Northern Europe, Austria or Switzerland ( $n$ 45), a high proportion was engaged in sports (73\%), while among women from Southern or Eastern Europe, including women from Turkey and Russia ( $n$ 65), only $34 \%$ were engaged in any sports. The proportion of women who usually did not use a bike was very high among women from Southern Europe (86\%), slightly lower among women from the USA or Northern Europe (69\%), followed by Eastern Europe (58\%) and Austria and Switzerland (37\%), which is comparable to German women (42\%). Our results are in line with a study from Sweden that found a higher likelihood for low LPA among immigrants from Southern and Eastern Europe ${ }^{(25)}$. A study in the USA reported that differences in educational attainment and health status accounted for virtually all of the racial and ethnic differences in $\mathrm{LPA}^{(26)}$. However, our result on non-German nationality persisted even after full adjustment. It may be worth considering how female immigrants, especially from Southern and Eastern Europe, including Turkey and Russia, could be reached by special sports, fitness or walking groups. In regions with larger immigrant communities, courses possibly could be offered in their native language.

Regarding marital status, the results are inconsistent. Some studies found higher activity among unmarried compared to married participants ${ }^{(10,12,13)}$, whereas other studies found lower activity among unmarried women ${ }^{(16,27)}$. The present study showed that widowed, separated and divorced women walked the most. The only two studies that investigated determinants for walking yielded similar results $^{(14,16)}$.

Interestingly, in our study, parity seemed to be more relevant than marital status for sports or cycling activity. Investigations of the influence of the number of children or age at first pregnancy on activity behaviour in later life are scarce. This is somewhat astonishing as children often substantially affect the lifestyle and recreational activities of their parents. To our knowledge, only two studies in younger women considered these aspects ${ }^{(16,23)}$. Both studies found that a smaller family size was related to higher activities in sports but fewer activities in the household. In contrast, we studied the long-term effects of parity, showing that later in life nulliparous women were less physically active than parous women. This was seen for singles as well as for ever married women who remained childless. Possibly, parous women were used to a more active life at younger ages, being responsible for children and a larger household, thus continue to be active at older ages through cycling and sports. Our finding that young age at first pregnancy was associated with a lower likelihood of being engaged in sports later in life, may reflect the reluctance to engage in sports in later adulthood when not used to doing sports in younger age, e.g. due to early maternity.

\section{Physical activity and coexisting diseases}

Women with previous benign breast disease, myoma or arthritis/rheumatism were slightly more likely to engage in sports. Only one study from China also reported higher sports activities of women following chronic disease diagnosis ${ }^{(16)}$. Otherwise, our results for medical conditions are in line with the most previous work indicating that poor health status is associated with decreased $\mathrm{PA}^{(10,15,28)}$. Our cross-sectional study cannot determine whether poor health resulted in decreased activity or poorer health was the result of previous low activity levels. However, the majority of reported coexisting diseases had been diagnosed several years before the interview, and excluding diagnoses within the previous two years did not change the results substantially. Independently of the other determinants, women with diabetes were more likely at a low activity level for 
sports, cycling and walking. Women diagnosed with CVD or hypertension engaged less in sports and cycling. Similar results were reported from non-type-specific, cross-sectional studies in the elderly from the UK, Australia, Canada and Greece $^{(13,15,29,30)}$. Women with a chronic disease reporting never engaging in any sports since the age of 50 years, accordingly did not exercise in the years after diagnosis. Thus, our results indicate that many women diagnosed with hypertension, diabetes or CVD did not engage in exercise post-diagnosis, although it is known that PA is beneficial for disease management. In our study, the diseases themselves have been named by some participants as barriers to being active. It has been considered to be risky to exercise under pre-existing diseases. This indicates that patients may need to be more strongly encouraged by their physicians to engage in adequate exercise in order to achieve the extensive benefits of PA for the management of these diseases ${ }^{(31)}$.

In summary, our study identified a number of individual subject-related factors associated with activity in sports, cycling or walking in women in Germany. Low levels of activity were found for overweight or obese women, fulltime employees, specific occupational groups, current smokers, immigrants, nulliparous women or women who got pregnant already at young age. In addition, women with CVD, hypertension or diabetes appeared to have comparably low levels of activity. Because of high and increasing prevalence of these diseases in the elderly and the corresponding high individual and public health burden, PA should be a major therapeutic strategy for medical care teams. Overall, the present study may have implications for preventive programmes as the results could help to define potential target groups for interventions as well as to specifically tailor PA enhancement programmes to the women's needs or personal situations.

\section{Acknowledgements}

The present study was supported by Deutsche Krebshilfe e.V., Grant no. 702892 BR I. The authors have no conflict of interest. K.S. contributed to the writing of the manuscript; J.C.-C. and D.F.-J., the principal investigators of the MARIE study, reviewed the manuscript; M.E.S. helped in the data analysis and manuscript writing. The authors are grateful to all study participants and to the interviewers who collected the data. The authors also thank T Slanger and $\mathrm{E}$ Mutschelknaus who coordinated the study in the two regions, and S Behrens, R Birr, W Busch, U Eilber, B Kaspereit, N Knese and K Smit for their most valuable technical assistance.

\section{References}

1. Eisenmenger M, Pötzsch O \& Sommer B (2006) Bevölkerung Deutschlands bis 2050 - 11. koordinierte Bevölkerungsvorausberechnung. Wiesbaden: Statistisches Bundesamt.
2. Bassuk SS \& Manson JE (2005) Epidemiological evidence for the role of physical activity in reducing risk of type 2 diabetes and cardiovascular disease. J Appl Physiol 99, 1193-1204.

3. Bonaiuti D, Shea B, Iovine R et al. (2002) Exercise for preventing and treating osteoporosis in postmenopausal women. Cochrane Database Syst Rev, issue 3, CD000333.

4. Hamer M \& Chida Y (2009) Physical activity and risk of neurodegenerative disease: a systematic review of prospective evidence. Psychol Med 39, 3-11.

5. World Cancer Research Fund/American Institute for Cancer Research (2007) Food, Nutrition, Physical Activity, and the Prevention of Cancer: A Global Prospective. Washington, DC: AICR.

6. Spirduso WW \& Cronin DL (2001) Exercise dose-response effects on quality of life and independent living in older adults. Med Sci Sports Exerc 33, S598-S608.

7. Gillespie LD, Gillespie WJ, Robertson MC et al. (2003) Interventions for preventing falls in elderly people. Cochrane Database Syst Rev, issue 4, CD000340.

8. Lampert T, Mensink GB \& Ziese T (2005) Sport and health among adults in Germany. Bundesgesundheitsblatt Gesundheitsforschung Gesundheitsschutz 48, 1357-1364.

9. Schneider S, Hauf C \& Schiltenwolf M (2005) Back care programs for health promotion-representative user profiles and correlates of participation in Germany. Prev Med 40, 227-238.

10. Becker S, Huy C, Brinkhoff KP et al. (2007) 'Living an active life' - sports, exercise and health in middle-aged and older adults. An empirical database on physical activity, health behavior and lifestyle in the 50- to 70-year-old residential population of Baden-Wuerttemberg. Gesundheitswesen 69, 401-407.

11. Schneider S \& Becker S (2005) Prevalence of physical activity among the working population and correlation with work-related factors: results from the first German National Health Survey. J Occup Health 47, 414-423.

12. Kaplan MS, Newsom JT, McFarland BH et al. (2001) Demographic and psychosocial correlates of physical activity in late life. Am J Prev Med 21, 306-312.

13. Pitsavos C, Panagiotakos DB, Lentzas Y et al. (2005) Epidemiology of leisure-time physical activity in sociodemographic, lifestyle and psychological characteristics of men and women in Greece: the ATTICA Study. BMC Public Health 5, 37-45.

14. Lawlor DA, Taylor M, Bedford C et al. (2002) Is housework good for health? Levels of physical activity and factors associated with activity in elderly women. Results from the British Women's Heart and Health Study. J Epidemiol Community Health 56, 473-478.

15. Harris TJ, Owen CG, Victor CR et al. (2009) What factors are associated with physical activity in older people, assessed objectively by accelerometry? Br J Sports Med 43, 442-450.

16. Jurj AL, Wen W, Gao YT et al. (2007) Patterns and correlates of physical activity: a cross-sectional study in urban Chinese women. BMC Public Health 7, 213-223.

17. Flesch-Janys D, Slanger T, Mutschelknauss E et al. (2008) Risk of different histological types of postmenopausal breast cancer by type and regimen of menopausal hormone therapy. Int J Cancer 123, 933-941.

18. Schmidt ME, Slanger T, Chang-Claude J et al. (2006) Evaluation of a short retrospective questionnaire for physical activity in women. Eur J Epidemiol 21, 575-585.

19. Ainsworth BE, Haskell WL, Whitt MC et al. (2000) Compendium of physical activities: an update of activity codes and MET intensities. Med Sci Sports Exerc 32, S498-S504.

20. Ainsworth BE, Haskell WL, Leon AS et al. (1993) Compendium of physical activities: classification of energy costs of human physical activities. Med Sci Sports Exerc 25, $71-80$. 
21. Schmidt ME, Steindorf K, Mutschelknauss E et al. (2008) Physical activity and postmenopausal breast cancer: effective periods in life and effect modification by different breast cancer characteristics. Cancer Epidemiol Biomarkers Prev 17, 3402-3410.

22. Atlantis E, Barnes EH \& Ball K (2008) Weight status and perception barriers to healthy physical activity and diet behavior. Int J Obes (Lond) 32, 343-352.

23. Sternfeld B, Ainsworth BE \& Quesenberry CP (1999) Physical activity patterns in a diverse population of women. Prev Med 28, 313-323.

24. Hillsdon M, Lawlor DA, Ebrahim S et al. (2008) Physical activity in older women: associations with area deprivation and with socioeconomic position over the life course: observations in the British Women's Heart and Health Study. J Epidemiol Community Health 62, 344-350.

25. Dawson AJ, Sundquist J \& Johansson SE (2005) The influence of ethnicity and length of time since immigration on physical activity. Ethn Health 10, 293-309.
26. He XZ \& Baker DW (2005) Differences in leisure-time, household, and work-related physical activity by race, ethnicity, and education. I Gen Intern Med 20, 259-266.

27. King AC, Kiernan M, Ahn DK et al. (1998) The effects of marital transitions on changes in physical activity: results from a 10-year community study. Ann Behav Med 20, 64-69.

28. Dishman RK, Sallis JF \& Orenstein DR (1985) The determinants of physical activity and exercise. Public Health Rep 100, 158-171.

29. Lim K \& Taylor L (2005) Factors associated with physical activity among older people - a population-based study. Prev Med 40, 33-40.

30. Plotnikoff RC, Taylor LM, Wilson PM et al. (2006) Factors associated with physical activity in Canadian adults with diabetes. Med Sci Sports Exerc 38, 1526-1534.

31. Kirk AF, Barnett J \& Mutrie N (2007) Physical activity consultation for people with type 2 diabetes: evidence and guidelines. Diabet Med 24, 809-816. 